\title{
Extending a flexible searching tool for multiple data- base formats
}

\author{
Mohammad Halim Deedar and Susana Muñoz Hernández \\ Campus de Montegancedo s/n, Escuela Técnica Superior de Ingenieros Informáticos, \\ Universidad Politécnica de Madrid, Boadilla del Monte 28660, Spain \\ halim.deedar@alumnos.upm.es, susana@fi.upm.es
}

\begin{abstract}
Although all databases frameworks let us make conventional (crisp) searches, there are only a few of them that take into account some flexible, fuzzy, expressive criteria. The few of them that provide some of these searching characteristics are restricted to a particular database format, as FleSe that is devoted to search in a flexible way over Prolog databases. We have extended FleSe framework by an extraordinary feature that allows users to query various conventional and modern databases such as Prolog, CSV, XLS, XLSX, MySQL, and MongoDB or JSON in a fuzzy way. We have developed an adaptable and configurable platform for it so that any user can personalize at runtime. The fuzzy searching criteria can be created and added in a very userfriendly way, so that any user can upload his/her conventional (crisp) database, define the fuzzy search criteria that he/she is interested in and search at the database flexibly and expressively using concepts as similarity, fuzziness, qualification, and negation.
\end{abstract}

Keywords: Fuzzy logic, Search engine, Databases, Database search framework.

\section{Introduction}

Databases store crisp (non-fuzzy) information but the way a user wants to retrieve information most of the time is not crisp, because their thoughts are usually more expressive (fuzzy). For example, if a user is looking for a "cheap", "rather cheap", or "not very expensive" restaurant in a database, it is not functional to have the information already stored with those fuzzy concepts. Searching in a fuzzy way not only gives us the exact information we are looking for, but it lets us to retrieve all the possible and available information very close to the criteria we have set for our query. For example, if we are looking for food with a lower price than $\$ 10$ in a restaurant database (having crisp information) and there is food whose price is $\$ 10.20$, so our query will not retrieve, even if it might be a reasonable price for us. Although there are tools devoted on accessing databases (containing crisp information) in a fuzzy way, such as the approach presented by P. Bosc and O. Pivert in [1], with the syntax identical to SQL which is a bit difficult for the non-expert users to understand as it 
requires advanced users to understand the syntax. FleSe was presented by V. PablosCeruelo and S. Muñoz Hernández in [2] which is a handy tool that mainly focused on providing flexible facilities for users in their fuzzy searching over Prolog databases. Its problem was that FleSe was constrained to search over Prolog databases. Considering those mentioned points, we present a framework that provides a simple syntax for any user to perform fuzzy or non-fuzzy query over any conventional and modern database formats (Prolog, CSV, XLS, XLSX, MySQL, and MongoDB or JSON) and we provide the users the facility of creating their fuzzy criteria effortlessly without having any knowledge about the mathematical syntax of fuzzy functions.

\section{RFuzzy}

RFuzzy [3] is a library implemented for Ciao Prolog [4] to increase the expressiveness of Prolog with the possibility of creating fuzzy information. The purpose of developing RFuzzy is mainly focused on reducing the complex syntax of Fuzzy Prolog [5], and to provide an easy way for representing a problem using fuzzy reasoning with real numbers. RFuzzy represents the truth values using real numbers, and the answers provided by the RFuzzy are never constraints, and it can differentiate between the crisp and fuzzy predicates without the interferences of the user, it can manage the introduction of truth values.

The points that make RFuzzy so distinctive are that RFuzzy can define types, and default values (general and condition). RFuzzy is the first tool that implemented multi-adjoint logic and with the help of that RFuzzy was able to represent the concept of credibility of the rules.

In general, RFuzzy contains the following features that make it different from other approaches:

- It represents the truth values using real numbers instead of unions of intervals between real number.

- It provides a concrete syntax to define types so that the user does not need to code them in Prolog.

- It gives direct results to the queries instead of constraints.

- It can implement both the default and partial default values.

- RFuzzy uses multi-adjoint logic [6] to model its semantic.

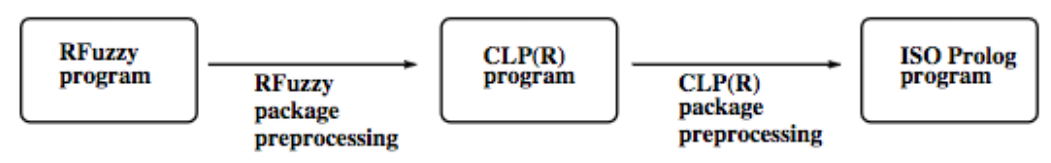

Fig. 1. RFuzzy architecture. 


\section{$3 \quad$ FleSe}

FleSe is a handy framework for performing fuzzy and non-fuzzy queries over Prolog databases containing crisp information. FleSe uses RFuzzy package which is a Prolog library developed for Ciao Prolog. FleSe uses fuzzy logic in a Prolog implementation. Fuzzy logic [7] assigns a membership degree to those elements which belong to a set. Therefore, FleSe has used this mechanism to perform the fuzzification of its searching criteria. Taking an example, a crisp database of restaurants (Table. 1) and the fuzzy definition of function "cheap" related to the average menu price as shown in Fig. 2 with the query of "Is there any cheap restaurant?", with the help of Fuzzy logic we obtain that Ni Hao is "Very" cheap, Tapasbar is "almost" cheap, II_tempietto is "hardly" cheap, and Kenzo is "not" cheap, and the usual answers for each individuals (very, almost, hardly, and not) are the truth values "1", "0.9", "0.2" and "0", respectively.

Table 1. Restaurant database.

\begin{tabular}{lll}
\hline Name & Price Average & Food type \\
\hline II_tempietto & 90 & Italian \\
Tapasbar & 20 & Spanish \\
Ni Hao & 10 & Chinese \\
Kenzo & 100 & Japanese \\
& & \\
\hline
\end{tabular}

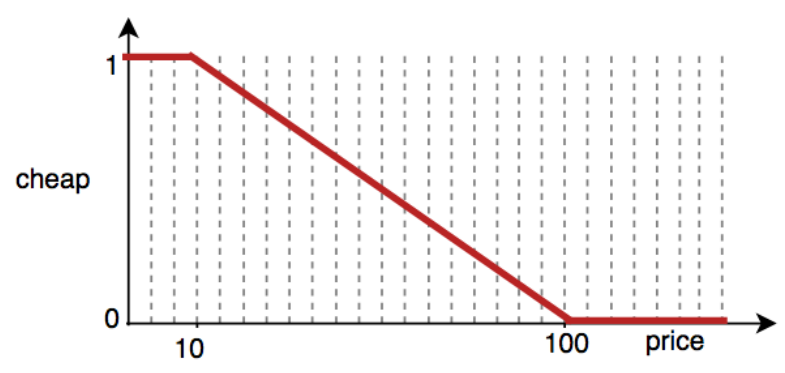

Fig. 2. Cheap fuzzification function.

\subsection{A brief review of semantics used by FleSe}

FleSe semantics are structured using multi-adjoint algebra [8], which enabled FleSe to get the credibility for the rules that we obtain from real-world data. Besides, the multi-adjoint algebra helped FleSe to create priorities for its rules, that it leads FleSe to obtain more useful and accurate result by getting the answers as a truth value with the maximum priority not the maximum value. 
The contribution of V. Pablos-Ceruelo and S. Muñoz Hernandez work in [9] based on multi-adjoint semantics provided a simple syntax and a complete semantics to the FleSe.

\subsection{Query Syntax}

FleSe gets the queries from users as input using the following syntax:

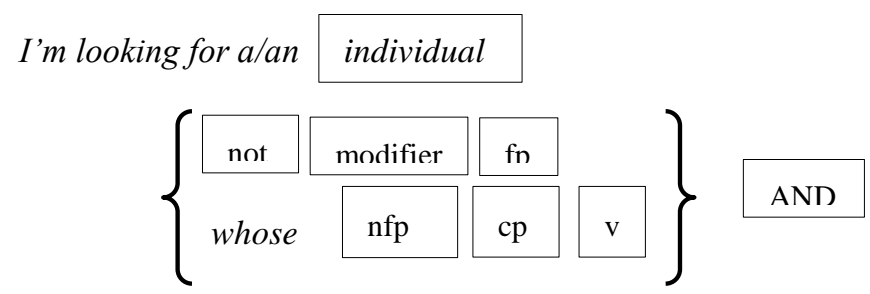

where:

- The individual represents the element we are looking for, such as (restaurant, film, car, ....).

- $\quad$ not gives a negation mechanism to the queries.

- $\quad$ modifier gives (quite, rather, very, ...) modifiers to the query.

- $f p$ stands for fuzzy predicates such as (cheap, large, close,...).

- $\quad n f p$ contains non-fuzzy predicates like (price, size, and distance to the center).

- $\quad c p$ stands for comparison operand, and it consists of ("is equal to", "is different from", "is bigger than", "is lower than", "is bigger than or equal to", is lower than or equal to "and "is similar to").

- $\quad v$ which stands for value, it asks for a crisp value when the users want to use the comp-op option such as ("is equal to", "is different from", or "is similar to"). The interface is more adaptable because the elements in the boxes can be modified and in case if we do not need any box we can leave it as blank.

- $A N D$ option will let the user add more options to the queries to make the query more efficient. Some examples to illustrate the syntax are: "I am looking for a film not very comedy" (Eq. 2), "I am looking for a film whose genre is romantic, and the duration is not more than 1 hour and 50" (Eq.3).

\begin{tabular}{|c|c|c|c|}
\hline \multirow[t]{2}{*}{ I'm looking for a/an } & film & & \\
\hline & not & very & comedy \\
\hline
\end{tabular}




\begin{tabular}{rl|l|l|l|l|}
\hline I'm looking for a/an & film & \multicolumn{2}{|l}{} \\
& whose & genre & is & romantic & AND \\
\cline { 2 - 4 } & whose & duration & is lower than & 50 minutes \\
\cline { 2 - 3 }
\end{tabular}

\subsection{New approaches added to FleSe}

The motivations of our work for introducing new approaches to FleSe includes the following points:

1- Database used by FleSe: FleSe allows the users to query only over Prolog databases, but the common databases that users are mostly dealing with are CSV, Excel and some modern databases such as MongoDB, MySQL, and so on.

2- The User-friendly interface of FleSe: The technical terms and some configuration options in the interface was not user-friendly enough for all types of users. Thus, our work devoted to the points as mentioned earlier includes:

- Now FleSe allows users to work with various database formats such as MongoDB, MySQL, XLS, XLSX, and CSV, and to perform expressive (fuzzy) searches over these databases.

- The interface is now more user-friendly having easy and non-technical terms for defining and searching any fuzzy or non-fuzzy criteria.

\section{$4 \quad$ Implementation details}

\subsection{Database uploading interface}

We developed the uploading interface of FleSe, which is a web interface written in Java, JavaScript, and HTML. The interface will let the databases of formats MongoDB, MySQL, CSV, XLS, XLSX, and Prolog to be uploaded into the framework as shown in Fig. 3. We provided a better uploading mechanism for the framework that other database formats can easily contribute with FleSe. The uploading interface includes a list of data-files, an option of sharing data-files with other users (Public or Private), and the option to remove uploaded data-files. The uploading process includes the following steps:

\section{Step 1: Parsing and conversion of databases into CSV}

The uploaded databases will get converted into Comma Separated Value (CSV) file after going through a parsing process of data-files. We made it so, that contents of the database files become more understandable and readable to the user, as there are some databases with a complex structure which are a bit difficult to be used by the user with less knowledge of databases such as syntax of MySQL, JSON, and so on. 


\section{Step 2: Defining column types}

Once the data-file has been converted into CSV (automatically by the system) the user can define the data-types for each column of the database as shown in Fig. [4] we have uploaded a database of cars having ten columns (id, manufacturer, class, year_of_production, horsepower, max_speed_in_kph, number_of_cylinder, fuel_tank_capacity, _fuel_type, price_in_euros) and then we have manually assigned the different data-types (enum_type, enum_type, enum_type, integer_type, integer_type, integer_type, integer_type, integer_type, enum_type, and integer_type.), respectively. After assigning the data-types, we need to click on "Create Prolog file" button to get the Prolog version of our database. The data-types offered by FleSe are boolean_type, enum_type, integer_type, float_type, and string_type. We provided an example of a film database with four columns in (Eq. 4) to show the syntax of RFuzzy database definition in FleSe.

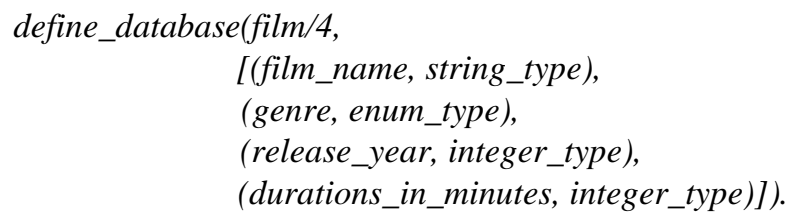

Each data-types defines the characteristics of a column and they help us while performing fuzzy or non-fuzzy searching over a database. For example, there are a set of comparison operators defined in FleSe available for each column based on their data types. The available comparison operators for integer_type and boolean_type are "is_equal_to" and "is_different_from", for enum_type the available comparison operators are "is_equal_to", "is_different_from", and "is_similar_to", and for string_type

$\&$ float_type the available comparison operators are "is_equal_to", "is_different_from","is_bigger_than",",is_lower_than","is_lower_than_or_equal_to", and "is_bigger_than_or_equal_to".

\section{Step 3: Converting CSV into Prolog}

Prolog is used for performing fuzzy searches in FleSe; Therefore, we have then converted our CSV intermediate data-file into a Prolog file; thus, our databases of any mentioned formats are ready for users in the system with the final format of Prolog database (containing crisp information) as shown in Fig. 5. 


\begin{tabular}{|c|c|c|c|c|}
\hline \multicolumn{3}{|c|}{ FleSe : Flexible Searches in Databases } & $\begin{array}{c}\text { logged as } \\
\text { halimdeedar_at_gmail_com_2_o } \\
\text { Data-files | Searching CCriteria | Personal Query }\end{array}$ & Sign out \\
\hline \multicolumn{2}{|c|}{ UPLOAD YOUR DATA FILES } & UPLOAD & & \\
\hline UPLOADED DATA FLLES & PRVIACTYY & REMOVE FLLE & & \\
\hline carsxis & O PUBUC & 而 & & \\
\hline film.sql & O pubuc & 西 & & \\
\hline restaurantison & O puвис & 西 & & \\
\hline shopping.csv & (1) pRIVATE & 面 & & \\
\hline sudents.xisx & O PRINATE & 而 & & \\
\hline travels.pl & O puBuc & 而 & & \\
\hline
\end{tabular}

Fig. 3. Database uploading interface.

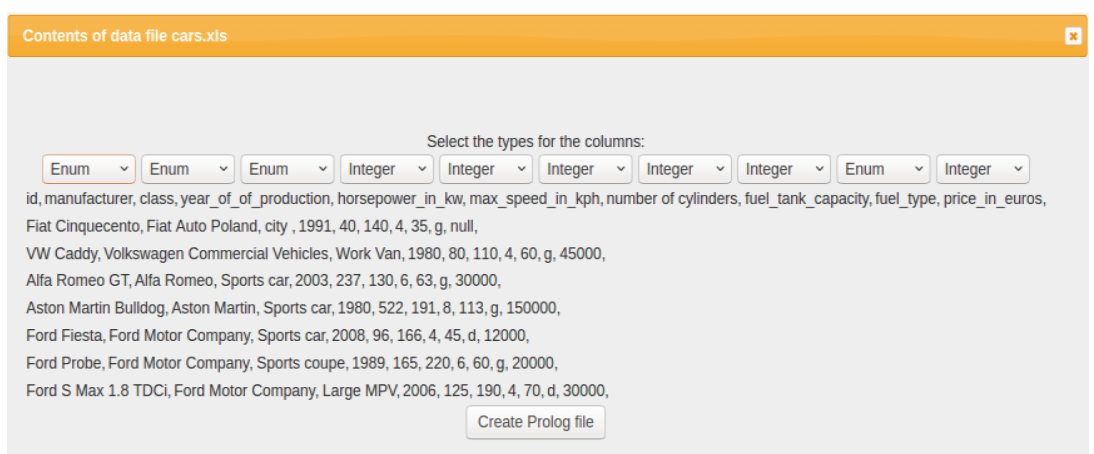

Fig. 4. Assigning data types for the column.

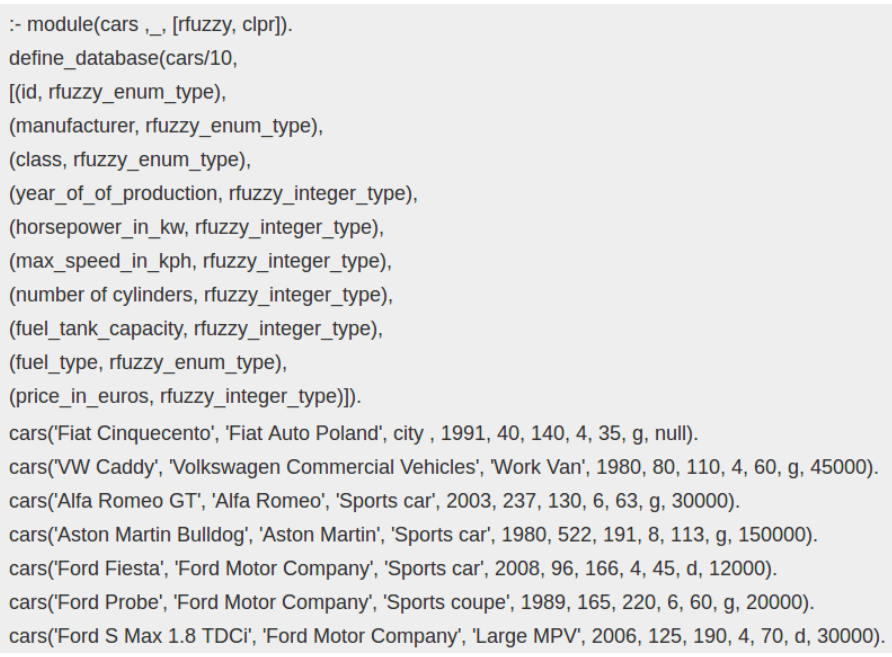

Fig. 5. Contents of Prolog data-file. 


\subsection{The Output Interface}

The answering interface (shown in Fig. 6) presents different sets of results in different tabs, the tabs with results in the best $30 \%$, the ones that satisfies the criteria more than the $50 \%$, the ones that satisfy the criteria in a (even small) degree and finally, all the results (the results whose truth value is over 0.7 , over 0.5 , over 0 and all the results) so that user can choose the one which satisfies his/her requirements. We used Ajax to prevent the wastage of computing time for getting the result form each tab.

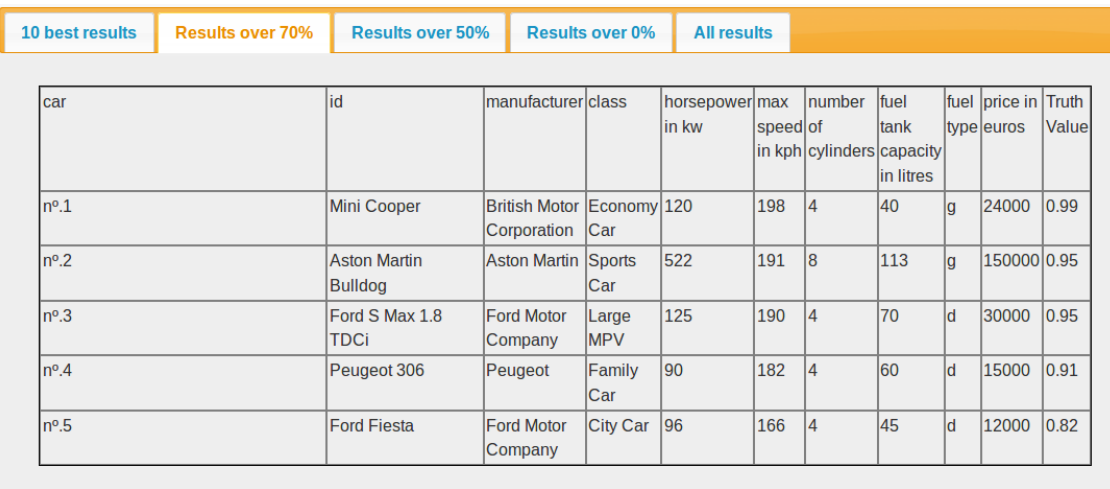

Fig. 6. Output interface.

We have asked the system for providing the fast cars from the Cars database that by the way was in XLS format. In Fig. 6 the result of our query is shown. The criterion of being fast is defined over a crisp characteristic of the car that is the speed. From the different tags that are offered by the output interface, we have chosen the one that is showing the $70 \%$ best results.

\section{Query Syntax}

\subsection{Combining crisp and fuzzy queries}

In FleSe we can execute a crisp and fuzzy query combined in a single query over a database of type SQL, JSON, XLS, XLSX, CSV, and Prolog. Taking an example of a film database with a query "Which drama films are not so long?" we can see that the first part of the query which is "looking for a drama film", is a crisp query and the second part of the query which is "a film with a not very long duration", is a fuzzy query therefore, FleSe allows us to combine and execute both the queries. We show in Fig. 7 how we can combine fuzzy and crisp query and the result is shown in Fig. 8. 


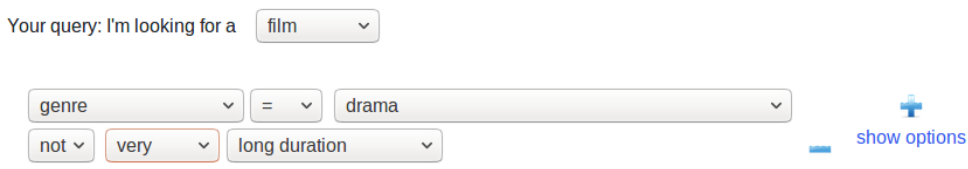

Fig. 7. Combining crisp and fuzzy queries.

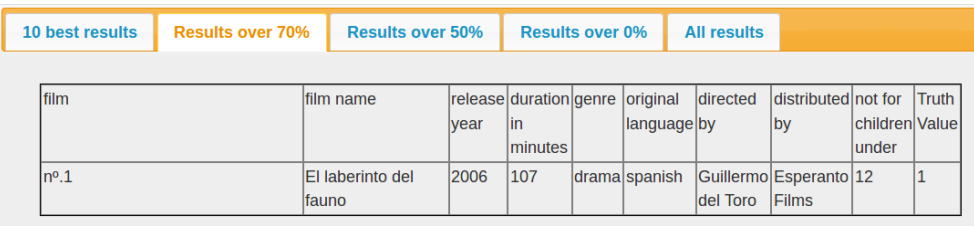

Fig. 8. Query result.

We can pose compound queries over various database formats such as SQL, JSON, CSV, XLS, XLSX, and Prolog. To clarify this, we take an example of a restaurant database with query "Which restaurants are costly, little traditional and rather close to the city center?". If we look to the query, in fact, it is three different fuzzy queries combined into a single query. Thus, FleSe allows us to create and execute such type of compound queries as shown in Fig. 9, we can see that the compound queries can be constructed using negation operator and quantifiers (little, rather, very) and the button ADD in the interface is helping us to add another condition or predicate in our query, and we have shown the result of our query in Fig. 10.

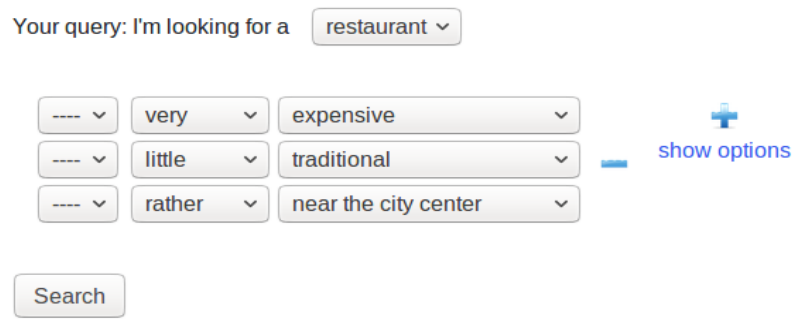

Fig. 9. Compound queries.

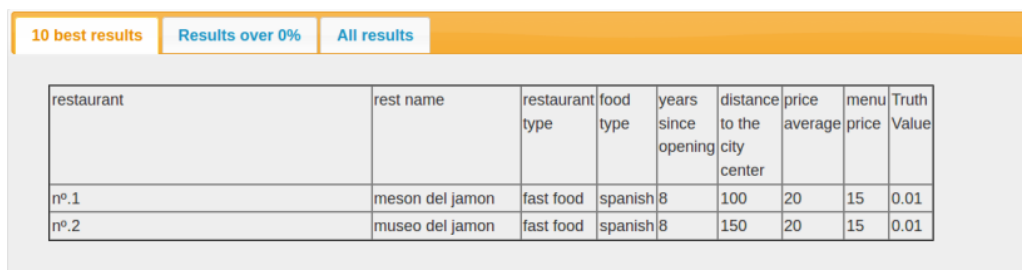

Fig. 10. Query result. 


\section{Conclusion}

It is always a challenge creating bridges between different fields to achieve join advances. There are some advances from fuzzy logic to provide expressive searching queries that use fuzzy criteria. But the promising prototypes are devoted to minority data formats as Prolog where most data owners lose the possibility to experiment with their data in the prototype. With this work, we have tried to reduce the gap between the database users and the logic programming users to let the first ones be able to search their crisp data in conventional formats in a flexible and expressive way. We have presented an advanced framework that allows a regular user to perform fuzzy and non-fuzzy queries over multiple database formats such as MongoDB, MySQL, CSV, XLS, XLSX, and Prolog with a very user-friendly querying interface (understandable by any non-expert users), and our framework is very adaptable that means any other database formats can easily incorporate with it. Being able construct simple queries to search in a flexible way by using expressive concepts as fuzzy criteria and similarity is a clear advantage of our framework with respect to any other standard available tool, such as MySQL. The purpose of our research is to present an intelligent searching system for databases that understand the queries from users and provide the results that he/she expects to obtain from any conventional and modern databases. Our current research focuses on the development of the framework allowing users to pose their expressive queries based on defining similarity relations criteria.

\section{References}

1. P. Bosc and O. Pivert, "SQLf: a relational database language for fuzzy querying", in IEEE Transactions on Fuzzy Systems, vol. 3, no. 1, pp. 1-17, Feb 1995, doi: 10.1109/91.366566.

2. V. Pablos-Ceruelo and S. Muñoz-Hernández, "FleSe: A Tool for Posing Flexible and Expressive (Fuzzy) Queries to a Regular Database", In Proc. Distributed Computing and Artificial Intelligence, 11th International Conference, 2014, pp.157-164.

3. S. Muñoz Hernández, V. Pablos-Ceruelo, and H. Strass, "RFuzzy: Syntax, Semantics and Implementation Details of a Simple and Expressive Fuzzy Tool over Prolog", Information Sciences, vol. 181, no. 10, pp. 1951-1970, 2011, doi $: 10.1016 /$ j.ins.2010.07.033.

4. The CLIP lab, "The Ciao Prolog Development System". [Online]. Available: http://www.clip.dia.fi.upm.es/Software/Ciao

5. S. Guadarrama, S. Muñoz, and C. Vaucheret, "Fuzzy prolog: a new approach using soft constraints propagation", Fuzzy Sets and System, vol. 144, no. 1, pp. 127150, 2004, doi: https://doi.org/10.1016/j.fss.2003.10.017.

6. V. Pablos-Ceruelo, H. Strass, and S. Muñoz Hernández, "RFuzzy-A framework for multi-adjoint Fuzzy Logic Programming", In Proc. Fuzzy Information Processing Society, 2009, pp. 1-6, doi:10.1109/NAFIPS.2009.5156427.LNCS.

7. L.A. Zadeh, "Fuzzy logic", Computer, vol. 21, no. 4, pp.83-93, 1988, doi $: 10.1109 / 2.53$ 
8. J. Medina, M. Ojeda-Aciego, and P. Vojtas, "A Procedural Semantics for Multiadjoint Logic Programming", In Proc. Progress in Artificial Intelligence, pp. 290297.

9. V. Pablos-Ceruelo and S. Muñoz Hernández, "Getting answers to fuzzy and flexible searches by easy modelling of real-world knowledge", In Proc. 5th International Joint Conference on Computational Intelligence, 2013, pp. 265-275, doi:10.5220/0004555302650272. 